hep-th/0508101

SINP/TNP/05-20

\title{
(A)symmetric Tachyon Rolling in de Sitter Spacetime: A universe devoid of Planck density
}

\author{
HARVENDRA Singh \\ Theory Division, Saha Institute of Nuclear Physics, \\ 1/AF Bidhannagar, Kolkata 700064, INDIA \\ [E-mail: h.singh@saha.ac.in]
}

\begin{abstract}
In a recent paper we studied rolling tachyon flat FRW cosmologies, but those admitting only time-reversal asymmetric boundary conditions. The time-reversal symmetric cosmologies have been studied by Sen previously. We show explicitly here that through appropriate choice of initial conditions, the time evolution of the Hubble parameter in these two types of solutions can be made completely identical for $t>0$, except near $t=0$. The rolling tachyon solution also gives rise to necessary inflation. We find that universe does start as a string size object (with string scale $10^{15} \mathrm{GeV}$ ) with a string mass density $\rho_{s} \simeq 10^{78} \mathrm{gm} . \mathrm{cm}^{-3}$ and not with Planck density.
\end{abstract}




\section{Introduction}

Recently, the phenomenon of tachyon condensation [1, 2] has been a subject of much attention in string theory and as well as in cosmological applications [3, 4. The tachyon field appears as instability in D $p$ - $\overline{\mathrm{D}} p$-branes or non-BPS D $p$-brane systems in type II string theory. ${ }^{1}$ The low energy dynamics of the open string tachyons living on the world-volume of unstable D3-brane is governed by the DBI action [7, 1, 2]

$$
-\int d^{4} x V(T) \sqrt{-\operatorname{det}\left(g_{\mu \nu}+\alpha^{\prime} \partial_{\mu} T \partial_{\nu} T\right)}
$$

where tachyon $T$ appears explicitly as a world volume scalar field, $g_{\mu \nu}$ represents the pullback of the spacetime metric. $V(T)$ is a positive definite tachyon potential which has a maximum while it vanishes as $T \rightarrow \infty$. The Regge slop parameter $\alpha^{\prime}=\left(l_{s}\right)^{2}=\left(M_{s}\right)^{-2}$ where $l_{s}$ is the string length and $M_{s}$ is the string mass. In a flat spacetime background the equation of motion for a purely time-dependent tachyon field assumes following unique form:

$$
\frac{V(T)}{\sqrt{1-\alpha^{\prime} \dot{T}^{2}}} \propto \rho .
$$

It means that the tachyon field behaves like a fluid of constant positive energy density, $\rho$, and of negative pressure, $P=-V \sqrt{1-\alpha^{\prime} \dot{T}^{2}}$. Indeed, if at the top of the potential $\dot{T}=0$, we have got an useful example of the cosmological constant for which $\rho=-P=$ constant. During the time evolution tachyon field rolls down the potential and reaches its true vacuum state given by $T=\infty$ where $V(\infty)=0$, while keeping $\rho$ constant through out. The pressure also vanishes in the tachyon vacuum. Thus the end state behaves like a pressureless matter (dust). When the tachyon system is coupled to nontrivial closed string background this result does get significantly modified and new type of cosmological solutions can be obtained. In recent times the application of open string tachyons in inflationary cosmological models has gained favourable attention; see the references [8, 9, 10, 11, 12, 13, 14, 15, 16, 17, 18, 19, 3, 4, 20, 24, 25. .

In the previous paper 24] we studied a very specific model in which tachyon action was coupled to de Sitter background. Especially we studied the flat homogeneous FRW cosmological solutions with time-reversal non-symmetric initial conditions. The solutions found there were completely non-singular and described inflationary situations just like after the big-bang. In this paper we would like to study the time-reversal symmetric model of Sen [3] and compare it to the time-reversal asymmetric situations in [24]. We find surprising similarities in the two cases. In particular we find it hard to distinguish, from the point in time where we are today, whether our universe has been time-reversal

\footnotetext{
${ }^{1}$ There are closed string tachyons in bosonic string theory as well; see [5] 6] for recent developments.

${ }^{2} \mathrm{~A}$ wide class of useful references on this topic can also be found in [3, 4, 21].
} 
symmetric or otherwise. We do also provide a detailed analysis of the inflation which has a greater appeal for phenomenological applications. The crucial point in our analysis is that we are specially considering a large number density of non-BPS branes over the compactified space. The large density of branes helps us in getting suitably high value of the Hubble constant during inflation. The previous attempts on inflationary tachyonic models however study the effects of spacetime warping on the tachyon potential; see [25] and references there in.

The paper is organised as follows. In section-2 we review and discuss the FRW cosmological models obtained by coupling bulk de Sitter supergravity to the tachyon action. We study the solutions both with and without time-reversal symmetric conditions. In section-3 we provide complete numerical analysis of simple inflationary models. We estimate the number of e-foldings during the inflation in realistic situations and fix the values of various parameters so that the results are close to the observed values. In section-4 we discuss the slow-roll limits coming from cosmology. We summarise the main results in section-5.

\section{2 (A)symmetric de Sitter cosmology with tachyon field}

We review the model considered by Sen [3] based on tachyon field theory coupled to background gravity and nonvanishing positive cosmological constant, $\Lambda$. It is significant in these models that compactification of string theory to four-dimensions with a positive cosmological constant, of KKLT type [22], is achieved. All the moduli including the volume modulus are taken to be apriori fixed. The model also has an unstable D3-brane which extends along the three large spatial directions. The four-dimensional effective action is taken as 3 ]

$$
S=\int d^{4} x\left[\frac{1}{16 \pi G} \sqrt{-g}(R-2 \Lambda)-V(T) \sqrt{-\operatorname{det}\left(g_{\mu \nu}+\alpha^{\prime} \partial_{\mu} T \partial_{\nu} T\right)}\right]
$$

where Newton's constant $G$ is related to four-dimensional Planck mass $M_{p}$ as $8 \pi G=M_{p}^{-2}$. We shall consider that at the top of the potential $V\left(T_{0}\right)=V_{0}$, while $V(\infty)=0$ in the tachyon vacuum. These are the two well known properties of the tachyon potential, e.g. $V(T)=V_{0} / \cosh (T / \sqrt{2})$ in superstring theory $\left[2 .{ }^{3}\right.$

The recent cosmological observations tell us that our universe is isotropic and homogeneous at large scales and also appears to be spatially flat [23]. These measurements

\footnotetext{
${ }^{3}$ Note that we shall take $V_{0} \sim N T_{3}$ for this potential, where $N$ is the number of 3-branes. We are considering more than one unstable 3 -brane or equivalently a fat 3 -brane. Here $T_{3}=\frac{\sqrt{2} M_{s}^{4}}{(2 \pi)^{3} g_{s}}$ is the non-BPS D3-brane tension.
} 
also reveal that the present phase of the expansion is such that the vacuum energy (the cosmological constant) contributes nearly $70 \%$ to the total energy density in the universe $\left(\Omega_{\text {total }}=1\right)$. Motivated by these phenomenological inputs we shall always assume $G V_{0} \gg \Lambda$. We look for purely time-dependent solutions of the equations of motion and shall take the metric ansatz to be a generic Friedman-Robertson-Walker spacetime

$$
d s^{2}=-d t^{2}+a(t)^{2}\left(\frac{d r^{2}}{1-k r^{2}}+r^{2}\left(d \theta^{2}+\sin ^{2} \theta d \phi^{2}\right)\right)
$$

where $k$ takes values 1,0 or -1 depending upon whether the spatial geometry is $S^{3}, R^{3}$ or $H^{3}$ respectively. We also take tachyon to be purely time-dependent $T=T(t)$.

With the above ansätze the field equations derived from the above action can be written as; the Tachyon equation:

$$
\ddot{T}=-\left(1-\alpha^{\prime} \dot{T}^{2}\right)\left(M_{s}^{2} \frac{V^{\prime}}{V}+3 \dot{T} \frac{\dot{a}}{a}\right)
$$

the Friedman equation:

$$
\frac{\ddot{a}}{a}=\frac{\Lambda}{3}+\frac{8 \pi G}{3}\left[\frac{V(T)}{\sqrt{1-\alpha^{\prime} \dot{T}^{2}}}\left(1-\frac{3}{2} \alpha^{\prime} \dot{T}^{2}\right)\right]
$$

and the Raychaudhuri equation:

$$
\left(\frac{\dot{a}}{a}\right)^{2}=-\frac{k}{a^{2}}+\frac{\Lambda}{3}+\frac{8 \pi G}{3} \frac{V(T)}{\sqrt{1-\alpha^{\prime} \dot{T}^{2}}}
$$

It can be seen that the equation (5) follows simply by taking the time derivative of the eq. (17). Therefore equations (6) and (7) contain all information about tachyon rolling in this model, making (5) redundant. Let us introduce a quantity $H(t) \equiv \dot{a}(t) / a(t)$ known as Hubble parameter at any given time $t$. Then equations (6) and (17) immediately give us

$$
\dot{H}=\frac{\kappa}{a^{2}}-\frac{3}{2} \frac{8 \pi G}{3} \frac{V(T)}{\sqrt{1-\alpha^{\prime} \dot{T}^{2}}} \alpha^{\prime} \dot{T}^{2}
$$

This is the key equation for the evolution of the Hubble parameter.

It can be seen that there is a time-reversal symmetry of these field equations if the fields (solutions) have the following properties [3]

$$
T(t)=T(-t), \quad H(t)=-H(-t), \quad a(t)=a(-t) .
$$

A generic time-reversal symmetric solution with boundary conditions 3 ]

$$
T(0)=T_{0}, \dot{a}(0)=0, a(0)=a_{0}, \dot{T}=0
$$


at $t=0$ necessarily requires $k=1$; that is, the spatial FRW geometry has to be $S^{3}$. However, for the time-asymmetric initial conditions [24]

$$
T(0)=T_{0}, H(0)=H_{0}, \dot{T}(0)=0
$$

at $t=0$ we can set $k=0$, which corresponds to a flat spatial geometry.

Note that the second term on the right hand side of (8) is always negative definite, while $\alpha^{\prime} \dot{T}^{2}$ can only vary between 0 and 1 monotonically. We clearly see from (8) , keeping in mind the conditions (10), that initially $\dot{H}>0$ where $\frac{k}{a^{2}}$ term is dominant while at the later stage the tachyon terms take over making $\dot{H}<0$ always. While specially for $k=0$ (time-asymmetric) case $\frac{d H}{d t}<0$ always [24]. Thus we also infer from here that the late time evolutions are the similar in the two cases, that is, at the later stage in tachyon evolution, Hubble constant always decreases with time or at most becomes constant (when $\Lambda \neq 0)$, but it cannot increase in time.

\section{$2.1 \mathrm{dS} \leftrightarrow \mathrm{dS}$ Vacuum Interpolation}

There are mainly two exact de Sitter solutions of the equations of motion in the last section with a constant tachyon field. The de Sitter solution with a bigger value of cosmological constant $\Lambda_{0}=\Lambda+8 \pi G V_{0}$ is given by [3]

$$
T=0, \quad \dot{T}=0, a(t)=a_{0} \cosh \left(\sqrt{\Lambda_{0} / 3} t\right), \quad a_{0}^{2}=3 / \Lambda_{0}
$$

and the other de Sitter solution is

$$
T=\infty, \quad \dot{T}=\frac{1}{\sqrt{\alpha^{\prime}}}, a(t)=a_{0} \cosh (\sqrt{\Lambda / 3} t), \quad a_{0}^{2}=3 / \Lambda
$$

Also, there will be other rolling tachyon solutions of the field equations which interpolate between these two de Sitter spaces. (Specially, if we set $\Lambda=0$, then the interpolation will be between dS and Minkowski flat spacetime.) This can be immediately seen over here. With the initial conditions (10), in the neighborhood of $t=0$ where $\dot{T} \simeq 0$, the field equations simply reduce to

$$
\begin{aligned}
& H^{2}=-\frac{\kappa}{a^{2}}+\frac{\Lambda}{3}+\frac{8 \pi G}{3} V_{0}+O\left(\alpha^{\prime} \dot{T}^{2}\right) \\
& \dot{H}=\frac{\kappa}{a^{2}}-O\left(\alpha^{\prime} \dot{T}^{2}\right)
\end{aligned}
$$

These are precisely the equations of a de Sitter spacetime with effective cosmological constant $\Lambda_{0}$. Hence the universe near the top of the potential inflates very fast as a de Sitter space. This pure de Sitter phase lasts for a very short time until $\dot{T}^{2}$ terms in (14) 
become sizable. We will also see from numerical analysis that in this short interval the Hubble parameter reaches its maximum value given by

$$
H_{m}=\sqrt{\frac{\Lambda_{0}}{3}}
$$

starting from the zero value. As $\dot{T}$ grows in time, from eq. (8), at some instant, $\dot{H}(t) \simeq 0$. While ever after that we will have decelerating (matter dominated) phase $\dot{H}<0$. In the far future (as $t \rightarrow \infty$ ) where $T \simeq \infty$ and $\alpha^{\prime} \dot{T}^{2} \simeq 1$, the field equations simplify to

$$
H^{2}=-\frac{\kappa}{a^{2}}+\frac{\Lambda}{3}, \quad \dot{H}=\frac{\kappa}{a^{2}}
$$

which is the de Sitter universe with a cosmological constant $\Lambda$. Thus the models with both the boundary conditions (10) or (11) will interpolate between two de Sitter phases with cosmological constants $\Lambda_{0}$ and $\Lambda$ respectively. The value of $\Lambda$ could as well be chosen to be the present value of cosmological constant in our universe, being $\sim 10^{-122} M_{p}^{2}$. To note, in the time-reversal symmetric case the Hubble parameter must change sign across $t=0$. Therefore universe must start with a (contracting) de Sitter phase at $t=-\infty$ and should end in the (expanding) de Sitter universe at $t=\infty$ separated by an intermediate de Sitter phase at $t=0$ [3].

\section{Numerical analysis of the tachyon rolling}

\subsection{Basic analysis}

An interpolating solution of the tachyon-gravity system as described in the last section will not be easy to obtain analytically. Therefore our main aim is to solve the tachyongravity system numerically. For simplification we set $\Lambda=0$, because for $\Lambda \ll G V_{0}$ the basic results will be similar except they will differ only at the late time. The equations (17) and (8) can be assembled in the following form

$$
\frac{\dot{H}-\frac{k}{a^{2}}}{H^{2}+\frac{k}{a^{2}}}=-\frac{3}{2} \alpha^{\prime} \dot{T}^{2}, \quad \frac{8 \pi G}{3} \frac{V(T)}{\sqrt{1-\alpha^{\prime} \dot{T}^{2}}}-\frac{k}{a^{2}}=H^{2} .
$$

First we are interested in the initial conditions (111) which represent the time asymmetric situation $(k=0)$ [24]. We take $T(0)=0, H(0)=H_{0}$ such that at the top of the potential $\dot{T}(0)=0$. Then eqs. (16) imply $\dot{H}(0)=0$ with $\frac{8 \pi G}{3} V(0)=H_{0}^{2}$. Classically the system with these initial conditions will not evolve in time, however a small fluctuation will dislocate the configuration from the top and the system will eventually start evolving. We shall take initial conditions in our numerical analysis in conformity with this fact 
where we are slightly away from the top position. We consider $V(T)=V_{0} / \cosh \left(\frac{T}{\sqrt{2}}\right)$ in these calculations for both symmetric and asymmetric cases. In our units $t$ is measured in $M_{p}^{-1}$ and the Hubble constant $H$ is measured in $M_{p}$. Accordingly the string tension parameter $\alpha^{\prime}$ which multiplies $\dot{T}^{2}$ has to be fixed. From string compactification

$$
M_{s}^{2}=\frac{g_{s}^{2}}{v_{0}} M_{p}^{2}
$$

with the volume parameter $v_{0} \equiv\left(\frac{R}{l_{s}}\right)^{6} \frac{1}{\pi}$ ( $R$ being radius of compactification). Since in this subsection, we are interested in the qualitative understanding of the tachyon rolling in symmetric and asymmetric cases, we just set $\frac{g_{s}^{2}}{v_{0}}=1$, so that $M_{s}=M_{p}$. Notice that $\frac{g_{s}^{2}}{v_{0}}=1$ corresponds to small volume compactification and so the results will be sensitive to stringy corrections. Therefore, for realistic scenarios we shall always consider large volume compactification in the next subsection.

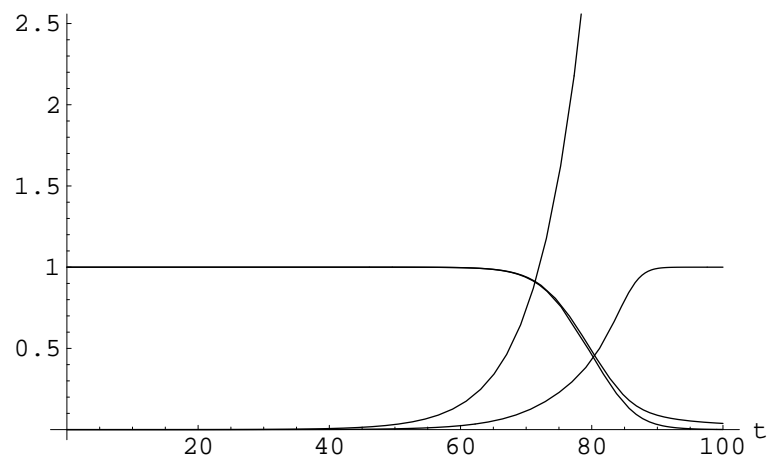

Figure 1: Plots are for the time evolution of T, $\dot{T}, H$ and $\sqrt{8 \pi G V / 3}$ presented in clockwise manner, with initial values $H(0)=1, T(0)=10^{-5}$. The value of $N_{e}$, which is the area under $H(t)$ curve in the plateau region, could easily be estimated to be close to 60. $H$ vanishes at late time but $V(T)$ vanishes faster than $H$.

The numerical results for two different time-asymmetric initial conditions (11) are plotted in the figures (11) and (2). Specially the time evolution of the various quantities $T, \dot{T}, H$ and $\sqrt{8 \pi G V(T) / 3}$ could be found in the figure (11). The plots of $H$ and $\sqrt{\frac{8 \pi G V}{3}}$ do overlap with each other in the plateau region. This plateau region we will characterise as the slow-roll inflationary period. Note that both $H$ and $V$ vanish at the late time, but 


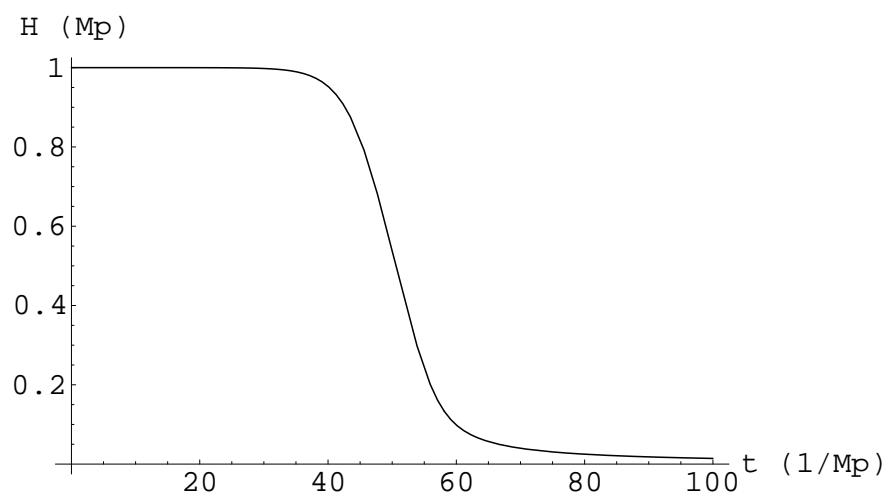

Figure 2: The graph is for $H$ with initial values $H(0)=1\left(M_{p}\right)$ and $T(0)=10^{-3}$. The value of $N_{e}$ can be estimated to be about 50 in this case. $H$ vanishes only at late time but earlier than in the previous graph.

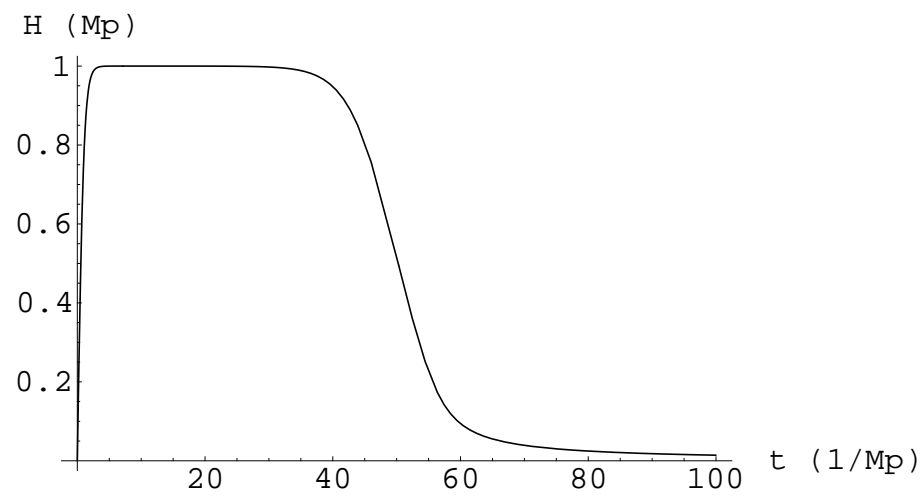

Figure 3: For comparison the plot is for $H(t)$ with time-reversal symmetric initial values $H(0)=0, T(0)=10^{-3}$ and $\frac{8 \pi G V(0)}{3}=1$. The value of $N_{e}$ is estimated to be 50 in this case. Note $H$ starts from initial value zero at $t=0$.

$V(T)$ vanishes faster than $H$. This fact led us to propose the bound ${ }^{4}$

$$
N_{e} \geq \int_{t_{i}}^{t_{f}} \sqrt{\frac{8 \pi G V}{3}} d t
$$

in the previous work [24]. From fig.(11), it is found that taking the initial values $H(0) \simeq$

\footnotetext{
${ }^{4}$ The number of e-foldings through which universe expands during the slow-roll inflation is defined as $N_{e}=\ln \frac{a\left(t_{f}\right)}{a\left(t_{i}\right)} \equiv \int_{t_{i}}^{t_{f}} H(t) d t$, where $t_{i}$ is the time when inflation starts and $t_{f}$ is the time when inflation ends. The estimate is that the universe went through at least 60 e-foldings.
} 
$1 M_{p}$ and the $T(0) \simeq 10^{-5}$ provide us with the number of e-folds during inflation close to 60. The duration of inflation is rather very short and is about $60\left(M_{p}\right)^{-1}$ only. But it can be increased by taking smaller values of $H(0)$ and $T(0)$, see [24]. At least from figures (11) and (2) it can be deduced that lower the initial value $T(0)$ longer is the duration of inflation.

Now we come to time-reversal symmetric evolution. We take the boundary conditions as in (10) along with $k=1$ in (16). The time evolution of $H(t)$ is plotted in fig [3] keeping the initial values $T(0)$ and $\frac{8 \pi G V(0)}{3}$ same as for fig 2. We can see from the figure (3) that near $t=0$ the Hubble parameter grows very-very fast starting from a initial zero value and in no time it reaches its maximum value of 1 . After that it remains constant for a while, this constant $H$ reason where $H$ is also maximum will be characterised as the slow-roll inflation. Later the matter dominated (decelerating) phase takes over which will terminate in a de Sitter phase as $t \rightarrow \infty$. Due to time-reversal symmetry, the plot must be extended to negative $t$ axis also, but we have not plotted it. However, the inflation lasts for a rather short interval of $50 M_{p}^{-1}$ only. Moreover, the value of the Hubble parameter at the end of inflation is of the order of $1 M_{p}$ which is much larger value than the COBE bound $10^{-5} M_{p}$ from the fluctuations. So these $H_{0} \sim O\left(M_{p}\right)$ models are not good and require a change in the various initial values of the parameters and fields.

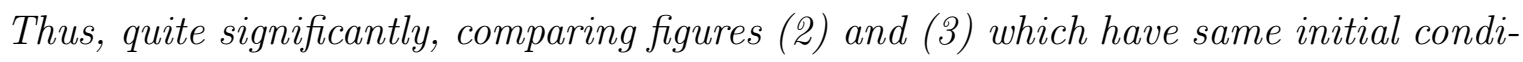
tions except for the value $\dot{a}(0)$, we learn that the time-symmetric and asymmetric evolutions of the Hubble parameter differ in picture only in the neighborhood of $t \simeq 0$, but not at later (positive) time. That is, by observing the universe at late times we may not be able to distinguish between the two situations.

\subsection{A Near Phenomenological Choice of Parameters}

The analysis of the last section looks very promising in that the tachyon rolling does seem to provide, albeit qualitatively, the picture of inflationary evolution of our universe. In order to get a realistic picture we need to pick those values of parameters $g_{s}, v_{0}, N$ which would restrict the size of perturbations $\delta_{H}$ (or density fluctuations) to less than $10^{-5} M_{p}$ towards the end of slow-roll inflation. This will mostly depend on the cosmological constant $\Lambda_{0}$ of the de Sitter phase near $t=0$ which controls the value of the Hubble parameter during inflation (the plateau region in the figures). The value of $\Lambda_{0}$ depends upon the height of tachyon potential through $\Lambda_{0}=\Lambda+8 \pi G V_{0}$. From the stringy origin of the tachyon potential, $V$, we find that the maximum value of Hubble parameter is (as $\left.\Lambda \ll 8 \pi G V_{0}\right)$

$$
\left(H_{m}\right)^{2}=\Lambda_{0} / 3 \simeq \frac{8 \pi G V_{0}}{3} \approx \frac{N}{g_{s}}\left(\frac{g_{s}^{2}}{v_{0}}\right)^{2} M_{p}^{2} .
$$


where $N$ counts the number of 3 -branes. ${ }^{5}$ To bring stringy corrections under control, we shall consider large volume compactification, $v_{0}>1$, with generic weak string coupling, $g_{s}<1$. Using eq.(17), the above equation (18) can also be written as

$$
H_{m} \sim \sqrt{\frac{N g_{s}}{v_{0}}} M_{s} .
$$

Thus, generically we will have a situation $H_{m}<<M_{s}<<M_{p}$ whenever $g_{S}<1$ and $v_{0}>1$. However, from our experience in the last section, for suitable inflation, cases with $H_{m} \simeq M_{s}$ are more favourable. From (19), this can be achieved only if we consider suitably large number of the branes so that $\frac{N g_{s}}{v_{0}} \sim 1$. In the following it will be our primary assumption.

Now, particularly if we take $g_{s}=0.1, v_{0}=10^{6}$, and $N / v_{0}=10$, then the string scale becomes $M_{s}=10^{-4} M_{p}$ and

$$
H_{m} \sim M_{s} \simeq 10^{-4} M_{p}
$$

While if we take $g_{s}=0.1$ and $v_{0}=10^{6}$, along with $N / v_{0} \sim 1$, the string scale is still $M_{s}=10^{-4} M_{p}$ but now

$$
H_{m} \sim 0.316 M_{s}
$$

Both of the situations (20) and (21) appear to be close to each other, but as we will see in next section that $H_{m}<M_{s}$ is less favoured for cosmological applications as this tend to spoil the slow-roll. So we shall take $H_{m} \sim M_{s} \sim 10^{-4} M_{p}$, i.e. (20), for the model which we are going to discuss in the rest of the paper. Also, keeping $H_{m} \sim M_{s}$ the closed string production rate during the tachyon decay will be rather suppressed (dilute approximation) compared to the situation when $H_{m} \gg M_{s}$. This may be important as we are assuming that classical action (3) remains valid through out the time evolution. Notice, however, taking $H_{m} \sim M_{s}$ also makes the temperature of the initial de Sitter phase comparable to the Hagedorn temperature of the strings. ${ }^{6}$

The Model:

Based on above approach, in the figure (4) we have plotted an interpolating (numerical) solution of the eqs.(16) by taking

$$
g_{s}=0.1, M_{s}=10^{-4} M_{p}, \quad 8 \pi G V_{0}=3 \times 10^{-8} M_{p}^{2}
$$

and the time-symmetric initial conditions $(k=1)$ at $t=0$ as

$$
T(0)=T_{0}=10^{-3}, \dot{a}(0)=0, a(0)=\sqrt{3 /\left(8 \pi G V_{0}\right)} .
$$

\footnotetext{
${ }^{5}$ Note we have dropped a factor of $\frac{\sqrt{2}}{3(2 \pi)^{3}}$ on the RHS of the above equation. Note, it can be easily absorbed in the parameter $N$ with out affecting the whole analysis.

${ }^{6}$ We are thankful to the referee for suggesting this important point. Nevertheless, we are assuming that the classical analysis holds good so long as $H \sim M_{s}$.
} 
This is just one of the suitable choices. One can take slightly different values of these quantities. For example, if we take $T(0) \sim .01$ keeping everything else same, the duration of inflation will be slightly reduced compared to the fig 4. We will discuss more on this in section-4. For practical reasons we have set $\Lambda=0$ while numerically solving the equations. Also if we keep $\Lambda \approx 10^{-122} M_{p}^{2}$ in these equations it will not alter the major conclusions.

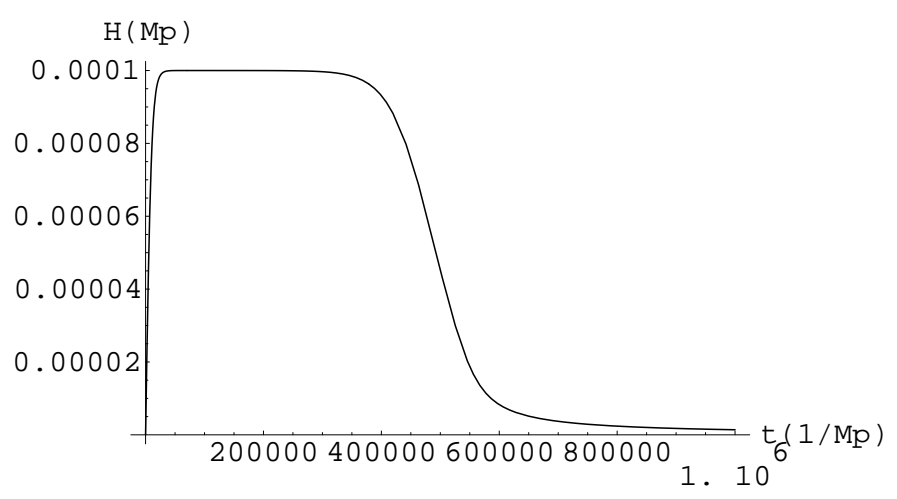

Figure 4: The plot is for $H(t)$ with time-reversal symmetric initial values $H(0)=$ $0, T(0)=10^{-3}$ with $\frac{8 \pi G V(0)}{3}=10^{-8}$. The value of $N_{e}$ could easily be estimated to be 50 in this case.

\section{Critical number density:}

It is important to emphasize that above construction heavily depends upon the proposal that we can have a large number of 3-branes such that $N / v_{0} \equiv N\left(\frac{l_{s}}{R}\right)^{6} \sim O(1)$. The quantity $N / v_{0}=1$ does quantify a critical number density for us. For example, a large compactification radius of $R=10 l_{s}$ will imply $N \sim 10^{6}$. Thus if compactification volume is large so is the number of branes, such that there is one 3-brane for each six-volume block (of string-size) of the compact six-manifold. If this flexibility is not there then we will not have the construction we discussed so far.

\subsection{Inflation and growth of scales}

It will be worth while to know, how the scales grow during the inflation and the rest of the expansion in the time symmetric model. The work is simplified if we divide the whole time evolution from $t=0$ to $t=t_{\text {today }}$ into definite intervals during which Hubble parameter grows differently. Based on the graph in fig 4 the evolution can be divided into four distinct phases of expansion. We shall use the values in eqs. (22), (23) and fig 4 for the estimate of various quantities in this section.

Phase-1 (pure de Sitter) 
During this phase $\dot{H}>0$, universe grows with an accelerated expansion from $t=0$ to the time $t_{i}$ where Hubble parameter reaches its maximum value $H_{m}=\sqrt{\Lambda_{0} / 3}$. This is a pure de Sitter phase during which scale factor grows as $a(t)=a_{0} \cosh \left(H_{m} t\right)$. Thus the expansion lasts typically for the period $\sim\left(H_{m}\right)^{-1}$. For our choice of $H_{m} \approx 10^{-4} M_{p}$ this period of de Sitter expansion is calculated to be around $10^{4}\left(M_{p}\right)^{-1}$. This is quite evident from the fig 4 also. The size of the universe grows roughly by 10 times of the initial size. The initial size at $t=0$ is, however, fixed by $a_{0}=\sqrt{3 / \Lambda_{0}}$, which is of the string size, $l_{s}$. From (22),$l_{s}$ is of the order of $10^{-29} \mathrm{~cm}$.

Phase-2 (Slow-roll inflation)

This phase has the typical characteristic that $\mathrm{H}$ varies very slowly $(\dot{H} \simeq 0)$. This corresponds to the plateau region in fig 4 . At the starting point $t_{i} \sim 10^{4} M_{p}^{-1}$ the value of Hubble parameter is $10^{-4} M_{p}$. It is clear that inflation ends at the time $t_{f} \sim 5 \times 10^{5} M_{p}^{-1}$ where $H \sim 10^{-5} M_{p}$. During this period the number of e-folds $N_{e} \simeq 50$ which is certainly less than the usual value of 60 e-folds. Thus the inflation ends early when universe is just about $10^{-37} \mathrm{sec}$ young. During this tiny interval scales grow by a factor of $e^{N_{e}} \simeq 10^{22}$, so the universe will grow into a size as big as $10^{-7} \mathrm{~cm}$ starting from the size $10^{-28} \mathrm{~cm}$ at the start of inflation.

Phase-3 (Matter dominated phase)

At the end of inflation, the matter content starts dominating the expansion and the Hubble parameter then evolves as $H(t) \propto \frac{2}{3}\left(\frac{1}{t}\right)[24$. This phase will last until $H \approx \sqrt{\Lambda / 3}$, where $\Lambda$ we would like to take as $10^{-122} M_{p}^{2}$, the observed value in our universe. From the simple relation

$$
\frac{H\left(t_{1}\right)}{H\left(t_{2}\right)}=\frac{t_{2}}{t_{1}}
$$

valid during this expansion we can estimate, taking $t_{1} \simeq 10^{-37} \mathrm{sec}, H\left(t_{1}\right) \simeq 10^{-5} M_{p}$, and $H\left(t_{2}\right) \sim 10^{-61} M_{p}$, that the age of universe is approximately $10^{11} \mathrm{yr}\left(1 \mathrm{yr}=3.15 \times 10^{7} \mathrm{sec}\right)$. Thus it gives more or less the correct age of our universe. Also for most of the time the universe has been dominated by matter phase. Using the scale factor growth as $a(t) \propto t^{2 / 3}$, we can estimate that size of the universe grows by a factor of $10^{38}$ during the matter phase. Accordingly the present size of our universe is calculated to be $\sim 10^{31} \mathrm{~cm}$, which is slightly larger than the present estimates on the size of the visible universe being $10^{29} \mathrm{~cm}$ [23, 26]. Phase-4 (de Sitter)

This is an expected end phase during which universe will keep on expanding forever like a de Sitter space with constant $H=\sqrt{\Lambda / 3} \sim 10^{-61} M_{p}$. This is one of the vacuum solution in the time (a)symmetric model.

Let us now plot the rate of change of Hubble parameter during the evolution of the universe. The graph in fig 5 corresponds to the initial values as in fig 4 . The behaviour of expansion resembles to our predictions in section-2. Initially $\dot{H}>0$, but then it starts to slow down and reaches the inflationary phase where $\dot{H} \sim 0$. It then further slows down 
and enters the region which is characterised by $\dot{H}<0$. The evolution then reaches close to the cusp region near about $t=500000 / M_{p}$ where $\ddot{H}=0$ briefly. This cusp region may be interesting for phenomenology, e.g. reheating of the universe etc. Beyond the cusp region $\dot{H} \propto-\frac{1}{t^{2}}$, which corresponds to the matter dominated era.

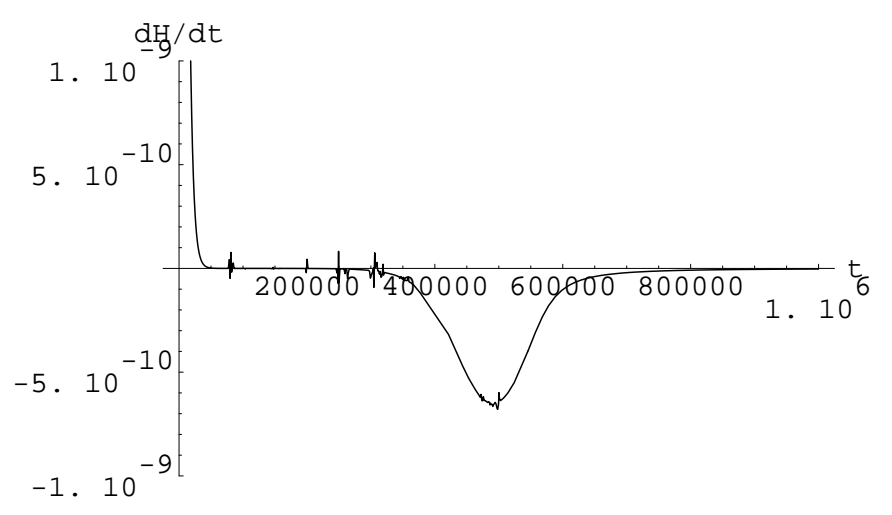

Figure 5: The plot is for $\dot{H}$ with time-reversal symmetric initial values.

In the end let us comment on the time-reversal asymmetric solution of the equations of motion. Looking at the figures (2) and (3) we find that except near $t \simeq 0$ the Hubble parameter evolves more or less in the similar fashion in the two models. But there will be no phase like $\dot{H}>0$ for the time-asymmetric case. A viable inflationary model with time-reversal asymmetric conditions will thus be as appealing as the one presented in this work and we will not attempt it separately.

\section{Slow-roll parameters and density perturbations}

Though it is quite evident from the previous analysis that there is genuine slow-roll inflation in the model. Let us find out the values of conventional slow-roll parameters in cosmology. The nearly scale invariant spectrum of the large scale structure in the universe provides two important conditions on the slow-roll parameters

$$
\epsilon \equiv \frac{M_{p}^{2}}{2 \alpha^{\prime}} \frac{V^{\prime 2}}{V^{3}} \ll 1, \quad \eta \equiv \frac{M_{p}^{2}}{\alpha^{\prime}}\left(\frac{V^{\prime \prime}}{V^{2}}-\frac{1}{2} \frac{V^{\prime 2}}{V^{3}}\right) \ll 1
$$

where derivatives are with respect to $T .^{7}$

\footnotetext{
${ }^{7}$ In the conventional form, for the Lagrangian with inflaton terms as $-\frac{1}{2} \partial_{\mu} \phi \partial^{\mu} \phi-V(\phi)$, the slow-roll parameters are given by $\epsilon \equiv \frac{M_{p}^{2}}{2} \frac{V^{\prime 2}}{V^{2}}, \eta \equiv M_{p}^{2} \frac{V^{\prime \prime}}{V}$. When compared to the tachyon field in our analysis a nontrivial field redefinition $\sqrt{\alpha^{\prime} V(T)} \partial T \equiv \partial \phi$ is required which gives the eqs.(24), see also [25].
} 
Let us evaluate these quantities for $V(T)=V_{0} / \cosh \left(\frac{T}{\sqrt{2}}\right)$, we get

$$
\begin{aligned}
& \epsilon=\frac{M_{s}^{2}}{8 \pi G V_{0}} \frac{\tanh ^{2}\left(\frac{T}{\sqrt{2}}\right) \cosh \left(\frac{T}{\sqrt{2}}\right)}{4} \\
& \eta=\frac{M_{s}^{2}}{8 \pi G V_{0}} \frac{\left(\frac{3}{2} \tanh ^{2}\left(\frac{T}{\sqrt{2}}\right)-1\right) \cosh \left(\frac{T}{\sqrt{2}}\right)}{2} .
\end{aligned}
$$

Thus the ratio $\frac{M_{s}^{2}}{8 \pi G V_{0}}$ is an important quantity in deciding the slow-roll. If we take

$$
\frac{M_{s}^{2}}{8 \pi G V_{0}} \sim O(1)
$$

that is what we have taken in the numerical analysis, see eq.(22), then during the plateau region where $T \ll \sqrt{2}$, it provides reasonably smaller values for the slow roll parameters. Substituting the values from equations (22) and (23) into eq.(25), it gives for $T \ll \sqrt{2}$

$$
\epsilon \simeq \frac{T^{2}}{24} \ll 1, \quad \eta \simeq-\frac{1}{6}
$$

This value of $\eta$ is comfortably small but is far away from $\eta<<1$ which is required for the scale invariance of density spectrum. ${ }^{8}{ }^{9}$ We remark here a few points.

- If we try to have $\frac{M_{s}^{2}}{8 \pi G V_{0}} \gg 1$, we are in grave danger of making $\eta \sim 1$ and spoiling the near scale invariance of the density spectrum.

- On the contrary, if we consider $\frac{M_{s}^{2}}{8 \pi G V_{0}} \ll 1$, we end up in the problem of having initial value of Hubble parameter much larger than the string scale. This choice will then require potential quantum corrections to be considered in any analysis.

As we just saw, taking $\frac{M_{s}^{2}}{8 \pi G V_{0}} \sim O(1)$ helps in a very subtle manner in producing slow-roll inflation.

The cosmological measurements also put an upper bound on the amplitudes of density perturbations during the slow-roll inflation. These perturbations grow in size during the inflation and during rest of later part the expansion. The COBE measurements give a bound on metric perturbations [27]

$$
\delta_{H} \equiv \frac{\sqrt{\alpha^{\prime}}}{\pi \sqrt{75} M_{p}^{3}} \frac{V^{2}}{V^{\prime}} \sim 2 \times 10^{-5}
$$

\footnotetext{
${ }^{8}$ Observationally, the spectral index of the density fluctuations, $n_{s}$, is found to be greater than .94 and therefore the relation $n_{s}-1 \simeq 2 \eta-6 \epsilon$ tells us that $\eta \leq .03$.

${ }^{9} \mathrm{I}$ am thankful to D. Ghoshal, S. Trivedi and A. Sen for prompting me to check out the possible error in the previous version of the paper.
} 
This bound is applicable nearly 60 e-folds prior to the end of inflation. We calculate

$$
\begin{aligned}
\delta_{H} & =\frac{\sqrt{2}}{\pi \sqrt{75}} \frac{8 \pi G V_{0}}{M_{s}^{2}} \frac{g_{s}}{\sqrt{v_{0}}} \operatorname{cosech}\left(\frac{T}{\sqrt{2}}\right) \\
& =\frac{3 \sqrt{2}}{\pi \sqrt{75}} 10^{-4} \operatorname{cosech}\left(\frac{T}{\sqrt{2}}\right) \\
& \approx \frac{6}{\pi \sqrt{75}} \frac{10^{-4}}{T} \quad \text { for } T \ll \sqrt{2}
\end{aligned}
$$

where we have used $M_{s}=\frac{g_{s}}{\sqrt{v_{0}}} M_{p}=10^{-4} M_{p}$ and the exact values in (22). Also note that from (23) the smallest value of $T$ is $T_{0}=10^{-3}$ in this model.

Here, it is significant to note that our chosen value $H \simeq M_{s}=10^{-4} M_{p}$ at the start of inflation is almost right. To see this we need to know the value of $T(t)$ at the time when fluctuations seed in. From the fig 4 , we only have about 50 e-foldings in total, hence the quantum fluctuations must seed in at the start of the inflation itself in order to comply with the COBE normalisations. At the beginning of the fluctuations it is reasonable to take $T \sim 10^{-2}$. From (29) this gives us

$$
\delta_{H} \sim 10^{-3}
$$

at the beginning of the last 50 e-folds. So there is slight problem with the COBE limits. The amplitudes we get are larger but those are not too far away from the observational limits.

We note that there is enough flexibility in the model in order to get $\delta_{H}$ right. For example, looking at (29) we find that a value of $\frac{g_{s}}{\sqrt{v_{0}}}$ less than $10^{-4}$ will be more favourable. It means, the string scale should be taken further small than $10^{-4} M_{p}$. Alternatively, the ratio $\frac{8 \pi G V_{0}}{M_{s}^{2}}$ which we have taken $\sim 1$ can be relaxed a bit and can be taken slightly greater than 1 . That is, $H_{m} / M_{s}$ should be slightly greater than 1 . This will allow a larger initial value of $T(0)$, while maintaining the same number of e-folds $N_{e} \sim 50$. However, $H_{m}>M_{s}$ might invite quantum stringy corrections as we stated before. Still a purely classical analysis may provide some useful insight. On the opposite side, from eq.(29) it will also be prudent to choose $8 \pi G V_{0}$ smaller than $M_{s}^{2}$, but it should be done only marginally, otherwise a large difference will interfere with slow-roll limits on $\epsilon$ and $\eta$. Particularly $\eta$ is very much sensitive to this. This last point corresponds to the choice in (21), which incorporates a critical number density of the branes. However, this later choice does not seem to be a better choice as it also has the potential to reduce the number of e-folds further down from 50. Amongst all of these, $H_{m}$ being slightly greater than $M_{s}$ is a better choice accompanied with a larger initial value of $T(0)$.

Thus any possible remedy of the large values of $\eta$ and $\delta_{H}$ has to necessarily involve taking $\frac{g_{s}}{\sqrt{v_{0}}}<10^{-4}$ and $H_{m}$ slightly greater than $M_{s}$ but not like $H_{m} \gg M_{s}$. In the next we demonstrate rolling solutions which include both of these conditions. 
Improved results with $\frac{g_{s}}{\sqrt{v_{0}}}<10^{-4}$ and $H_{m}>\sim M_{s}$ : We basically present two examples which incorporate $\frac{g_{s}}{\sqrt{v_{0}}}<10^{-4}$ and $H_{m}>M_{s}$.

Example-1: Let us consider taking the parameters as

$$
\frac{g_{s}}{\sqrt{v_{0}}}=10^{-5}, M_{s}=10^{-5} M_{p}, \quad 8 \pi G V_{0}=9 \times 10^{-10} M_{p}^{2}
$$

with the time-symmetric initial conditions at $t=0$ as

$$
T(0)=10^{-2}, \dot{a}(0)=0, a(0)=\sqrt{3 /\left(8 \pi G V_{0}\right)} .
$$

That is, the string scale is $M_{s}=10^{14} \mathrm{GeV}$ and $H_{m}=\sqrt{3} M_{s}$. Note we can still trust a purely classical analysis. We have plotted the result in the figure (6) for the rolling solution of eqs.(16]) with the above initial conditions.

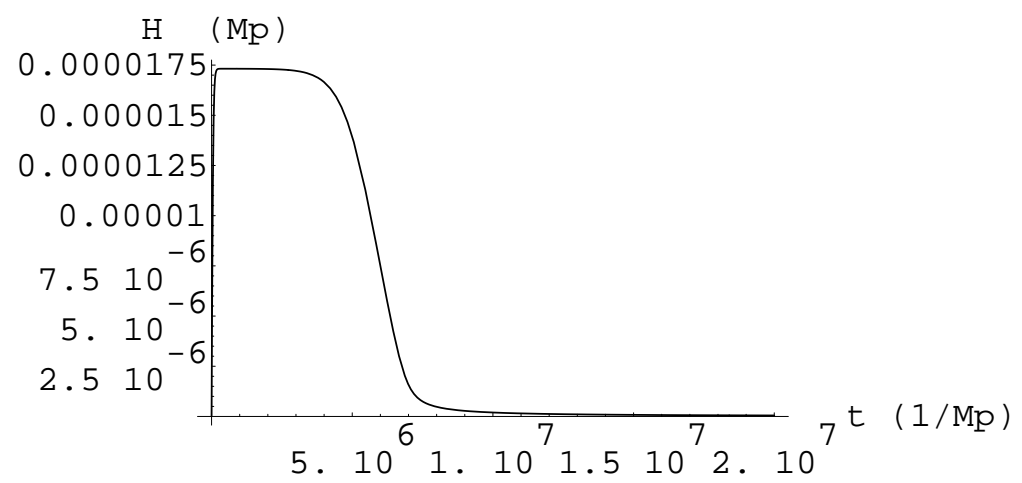

Figure 6: The plot is for $H(t)$ with time-reversal symmetric initial values $H(0)=$ $0, T(0)=10^{-2}$ with $\frac{8 \pi G V(0)}{3}=3 \times 10^{-10}$. The value of $N_{e} \sim 85$.

With this parametric choice we find that $\eta \simeq-\frac{1}{18}$, which gives $n_{s}=0.89$ and thus is much suitable for slow-roll inflation compared to the value of $\eta \simeq-\frac{1}{6}$ and may produce scale-invariant density spectrum. For the size of the amplitudes at 60 e-folds prior to the end of inflation, we now find that

$$
\left.\delta_{H}\right|_{60 e-\text { folds }} \simeq 1.3 \times 10^{-4},
$$

which is only marginally larger than the cosmological limits. This shows that there is definite improvement in the over-all picture.

Example-2: Let us now consider another set of values

$$
\frac{g_{s}}{\sqrt{v_{0}}}=10^{-5}, M_{s}=10^{-5} M_{p}, \quad 8 \pi G V_{0}=12 \times 10^{-10} M_{p}^{2}
$$


with the same initial conditions as in (31). The string scale is still $M_{s}=10^{14} \mathrm{GeV}$ but $H_{m}=2 M_{s}$. We find that this model gives $\eta \sim-\frac{1}{24}$ and the amplitudes as

$$
\left.\delta_{H}\right|_{60 e-\text { folds }} \simeq 2.6 \times 10^{-5}
$$

which is what one requires for the phenomenology.

Thus, essentially we have discovered that a string mass scale as low as $10^{14} \mathrm{GeV}$ together with a maximum value of Hubble parameter in the range $2 M_{s} \geq H_{m} \geq \sqrt{3} M_{s}$ can produce the desired slow-roll results within the reach of observational bounds on $\epsilon, \eta$ and $\delta_{H}$.

\section{Conclusions}

We have studied rolling tachyon cosmological solutions in a de Sitter spacetime. The Friedman-Robertson-Walker spacetimes are studied with both time-reversal symmetric as well as non-symmetric initial conditions. As stated by Sen [3] the time-symmetric conditions are the ones which are favoured by the open-string completeness conjecture. Although, the time asymmetric conditions are equally allowed but the corresponding open-string picture is not quite understood. From our study of the FRW solutions of the equations of motion, we find that from phenomenological point of view it is hard to distinguish between the two type of situations. Particularly, given the tachyon potential, the evolution of the Hubble parameter is strikingly similar except near $t=0$, in both the situations. The configurations are completely non-singular in both the cases.

In order to get a viable cosmological models of inflation, we need to have at least $8 \pi G V_{0} \simeq M_{s}^{2}$ with $M_{s} \simeq 10^{15} \mathrm{GeV}$ or lower. With large volume compactification $\left(v_{0}>1\right)$, this construction heavily depends upon a large number density of unstable 3-branes over the six-volume of the compact manifold. This is because we must have $\frac{N g_{s}}{v_{0}} \sim O(1)$ in order that $H_{m} \sim M_{s}$, which helps in achieving inflation. We define a critical number density for which there is single 3-brane per unit (string-size) six-volume of the compact manifold.

We find that near $t=0$ the universe will have an energy density of the order of $10^{78} \mathrm{gm} . \mathrm{cm}^{-3}$ and not the Planck density $10^{94} \mathrm{gm} \cdot \mathrm{cm}^{-3}$ as in big-bang scenario. The reason is that we cannot afford to have Planck density in these models at $t=0$, if we want appropriate amplitudes for the density fluctuations during the inflation. In the section-3, we have analysed a particular model in detail where solutions give rise to roughly 50 e-folds of inflation. Due to the restricted number of e-folds in this model, the fluctuations which give rise to the density perturbations, must set in at the very beginning of the inflation. Note that, during inflation the energy density of universe remains almost fixed. This is because the inflation is largely driven by the initial de Sitter like phase. Therefore at the 
time $t \simeq 10^{-37} \mathrm{sec}$ when inflation ends we will measure $H \sim 10^{14} \mathrm{GeV}$ with an energy density approximately $10^{78} \mathrm{gm} . \mathrm{cm}^{-3}$. These are more or less the standard values at the end of inflation in standard big-bang model. After the inflation the matter dominated phase starts which lasts until when $H \approx 10^{-61} M_{p}$.

We also find that during the large part of its evolution the universe has remained matter dominated. The calculated age of universe comes out to be about $\sim 10^{11} \mathrm{yr}$ which is quite admissible. Also the size of visible universe comes out to be $\sim 10^{30} \mathrm{~cm}$, which is just right in magnitude when compared to cosmological data. What about the radiation dominated phase? The answer is; we do not explicitly encounter a radiation dominated era where scale factor would grow as $a(t) \propto t^{\frac{1}{2}}$. But since the expansion switches in time from $\dot{H}>0$ (early de Sitter phase) to $\dot{H} \propto-\frac{2}{3} t^{-2}$ (matter dominated phase) through an intermediate value $\dot{H} \simeq 0$ (inflationary phase), somewhere in between (just after the inflation) the universe may pass through radiation dominated era.

In summary, it seems that inflationary tachyon models of the type presented in this work may be suitable for cosmological applications. We have found from our analysis that having string mass scale as low as $10^{14} \mathrm{GeV}$ together with $2 M_{s} \geq H_{m} \geq \sqrt{3} M_{s}$ does produce the desired slow-roll results within the observational limits on $\epsilon, \eta$ and $\delta_{H}$. However, there are limitations. These models require large number density of the non-BPS branes. Whether it can be admitted is an open question. From string theory point of view such a construction does provide a viable alternative to those models which assume spacetime warping [25]. In cosmology, particularly, we have to ask an important question; Is it that our universe never had a Planck density phase? Can we do without Planck density phase at the big-bang in cosmology? The scenarios we discussed in this paper do not support a Planck density phase. However, we do not claim to know the complete answer.

\section{Acknowledgment:}

I would like to thank Shibaji Roy for careful reading of the manuscript and for the useful discussion that followed. I am extremely thankful to the referee for the comments which have resulted in the major improvements of this manuscript.

\section{References}

[1] A. Sen, Rolling Tachyon, JHEP 0204 (2002) 048, hep-th/0203211.

[2] A. Sen, Tachyon Matter, JHEP 0207 (2002) 065, hep-th/0203265.

[3] A. Sen, Remarks on Tachyon Driven Cosmology, hep-th/0312153.

[4] A. Linde, Inflation and string Cosmology hep-th/0503195. 
[5] M. Headrick, S. Minwalla and T. Takayanagi,Closed string tachyon condensation: An overview, Class. Quan. Grav. 21 (2004) S1539, hep-th/0405064.

[6] H. Yang and B. Zweibach, Rolling Closed String Tachyons and Big Crunch, hep-th/0506076.

[7] A. Sen, JHEP 9910 (1999) 008, hep-th/9909062; M.R. Garousi, Nucl. Phys. B584 (2000) 284, hep-th/0003122; E.A. Bergshoeff, M. de Roo, T.C. de Wit, E. Eyras and S. Panda, JHEP 0005 (2000) 009, hep-th/0003221; J. Kluson, hep-th/0004106; D. Kutasov and V. Niarchos, hep-th/0304045.

[8] A. Mazumdar, S. Panda and A. Perez-Lorenzana, Nucl. Phys. B 614, 101 (2001) arXiv:hep-ph/0107058.

[9] G W Gibbons, Cosmological Evolution of the Rolling Tachyon, hep-th/0204008.

[10] M. Fairbairn and M.H. Tytgat, hep-th/0204070.

[11] D. Choudhury, D. Ghoshal, D.P. Jatkar and S. Panda, hep-th/0204204.

[12] L. Kofman and A. Linde, Problems with Tachyon Inflation, hep-th/0205121.

[13] M. Sami, P. Chingangbam and T. Qureshi, Phys. Rev. D66 (2002) 043530, hep-th/0205179.

[14] I. Y. Aref'eva, L. V. Joukovskaya and A. S. Koshelev, JHEP 0309, 012 (2003) arXiv:hep-th/0301137.

[15] F. Leblond and A.W. Peet, JHEP 0304 (2003) 048, hep-th/0303035.

[16] S. Roy, Phys. Lett. B567 (2003) 322, hep-th/0304084; N. Ohta, Phys. Rev. Lett. 91 (2003) 061303, hep-th/0303238.

[17] S. Nojiri and S.D. Odintsov, hep-th/0306212.

[18] S. Kachru, R. Kallosh, A. Linde, J. Maldacena, L. McAllister and S.P. Trivedi, hep-th/0308055.

[19] M. Garousi, M. Sami and S. Tsujikawa, Phys. Rev. D70 (2004) 043536, hep-th/0402075.

[20] A. Linde, Particle Physics and Inflationary Cosmology, (Harwood, Chur, Switzerland, 1990); hep-th/0503203. 
[21] T. Padmanabhan, gr-qc/0503107; J.S. Bagla, H.K. Jassal and T. Padmanabhan, astro-ph/0212198.

[22] S. Kachru, R. Kallosh, A. Linde and S.P. Trivedi, Phys. Rev. D68 (2003) 046005, hep-th/0301240.

[23] U. Seljak, astro-ph/0407372 C.L. Bennett et al., Astrophys. J. Suppl, 148 (2003) 1, hep-th/0302207; D.D. Spergel et al., Astrophys. J. Suppl, 148 (2003) 175, hep-th/0302209.

[24] H. Singh, More on Tachyon Cosmology in de Sitter Gravity, hep-th/0505012.

[25] D. Cremades, F. Quevedo and A. Sinha, Warped Inflation in Type IIB Flux Compactifications and the Open-String Completeness Conjecture, hep-th/0505252.

[26] See for example; J.B. Hartle, Gravity (Pearson Education, Indian print, 2003); S. Weinberg, Gravitation and Cosmology, (Wiley \& Sons)

[27] A.R. Liddle and D.H. Lyth, Cosmological inflation and Large Scale Structure, Cambridge University Press (2000). 